
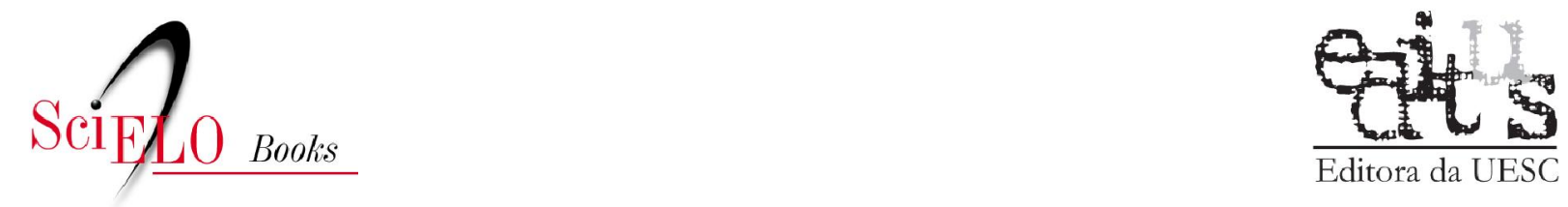

Editora da UESC

\title{
8 - Filogenia e sistemática das formigas poneromorfas
}

\author{
Fernando Fernández \\ John E. Lattke
}

FERNÁNDEZ, F., and LATTKE, JE. Filogenia e sistemática das formigas poneromorfas. In: DELABIE, JHC., et al., orgs. As formigas poneromorfas do Brasil [online]. Ilhéus, BA: Editus, 2015,

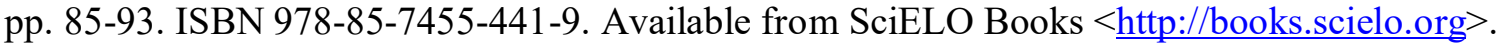

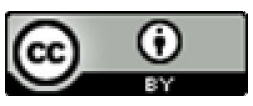

All the contents of this work, except where otherwise noted, is licensed under a Creative Commons Attribution 4.0 International license.

Todo o conteúdo deste trabalho, exceto quando houver ressalva, é publicado sob a licença Creative Commons Atribição 4.0.

Todo el contenido de esta obra, excepto donde se indique lo contrario, está bajo licencia de la licencia Creative Commons Reconocimento 4.0. 


\section{Filogenia e sistemática das formigas poneromorfas}

Fernando Fernández, John E. Lattke

\section{Resumo}

As formigas da subfamília Ponerinae, em seu senso original, possuem um longo e complexo histórico taxonômico que revisamos neste capítulo. Por muitos anos (de 1950 aos anos 1990), seguindo a proposta de William Brown Jr., estas formigas foram consideradas uma única subfamília, que abrangia uma série de subfamílias atuais dos presentemente reconhecidos grupo poneroide e clado formicoide. Nos anos 1990, Barry Bolton propôs novas interpretações sobre a classificação do grupo com base na morfologia do pecíolo e gáster destas formigas, e separou Cerapachyinae de Ponerinae. Em 2003,
Bolton produziu uma reclassificação das formigas e dividiu Ponerinae em várias subfamílias, coletivamente nomeadas "poneromorfas". Contudo, após 2006, diferentes filogenias utilizando ferramentas moleculares mostraram que as "poneromorfas" seriam um grupo parafilético e que dois de seus componentes, Heteroponerinae e Ectatomminae, seriam membros do clado formicoide, provavelmente próximos à Myrmicinae. Assim, Ponerinae em seu senso abrangente e antigo inclui diversas linhagens de formigas que provavelmente evoluíram convergentemente a partir do Paleoceno.

FERNÁNDEZ, F.; LATTKE, J. E. Filogenia e sistemática das formigas poneromorfas. In: DELABIE, Jacques H. C. et al. As formigas poneromorfas do Brasil. Ilhéus: Editus, 2015. p. 85-93. 


\section{Abstract}

Phylogeny and systematics of the poneromorph ants - Ants in the original subfamily Ponerinae, have had a long and complex taxonomic history, which we review in this chapter. For many years (from the 50's to the 90's), and following the proposed reclassification of William Brown Jr., these ants were considered as a single subfamily, which included several current subfamilies of the currently recognized poneroid group and formicoid clade. By 1990, Barry Bolton proposed new interpretations based on the morphology of the petiole and gaster of these ants and separated Cerapachyinae from the Ponerinae. In 2003, Barry Bolton produced a reclassification of ants and split the Ponerinae into several subfamilies, collectively referred to as "poneromorphs". However, after 2006, several phylogenies using molecular approaches have shown that "poneromorphs" are probably a paraphyletic clade and two of its components, Heteroponerinae and Ectatomminae, are members of a formicoid clade, probably close to Myrmicinae. Therefore, Ponerinae in the broad and early sense belong to several lineages, which probably evolved convergently from the Paleocene times onwards.

\section{Introdução}

Como um dos grupos mais importantes de insetos, as formigas (Hymenoptera: Formicidae) têm sido objeto de numerosos estudos que pretendem elucidar se este é um grupo natural (monofilético), sua posição entre os himenópteros e suas relações internas. Nas últimas décadas, avanços notáveis foram feitos nestes três aspectos, ainda que tenhamos muitos problemas para resolver. Neste capítulo exploramos a composição, filogenia e relações das formigas anteriormente chamadas "poneromorfas" (BOLTON, 2003) com outros grupos de formigas.

A monofilia das formigas nunca foi questionada e há muito tempo este é considerado um grupo natural, claramente separado dos demais himenópteros. A presença da glândula metapleural é uma das características únicas das formigas e tem sido considerada a sinapomorfias morfológica por excelência, sendo visível inclusive em alguns fósseis em âmbar (GRIMALDI; AGOSTI, 2000) com quase 100 milhões de anos de idade. A presença de um pecíolo, antenas geniculadas, saco infrabucal, asas decíduas nas fêmeas reprodutoras e colônias acelulares perenes com operárias (fêmeas estéreis sempre ápteras) são caracteres adicionais e únicos em Hymenoptera (BOLTON, 2003; TAYLOR, 2007).

Qual é a posição das formigas em Hymenoptera? Está claro que pertencem ao clado dos himenópteros com ferrão (Aculeata), um grupo monofilético cujas fêmeas possuem um ferrão modificado a partir do ovipositor, comum em grupos de vespas. Nas propostas filogenéticas mais recentes, Aculeata aparece em um clado que inclui Evanioidea, Ceraphronoidea e Trigonalioidea (HERATY et al., 2011). Dentro de Aculeata, as formigas são posicionadas próximo aos vespoideos, seja como uma família dentro deste grupo ou como sua própria superfamília. As relações internas de Aculeata não estão bem definidas, ainda que haja consenso em considerar Chrysidoidea como o grupo irmão do resto de Aculeata (BROTHERS; CARPENTER, 1993). A filogenia de Aculeata proposta por Brothers (1975) é a que tem sido aceita há mais tempo (BROTHERS; CARPENTER, 1993; RONQUIST et al., 1999), com a proposta Chrysidoidea + (Apoidea + Vespoidea). Ainda que haja sinapomorfias para as primeiras duas superfamílias, o mesmo não pode ser dito para Vespoidea. $O$ prepecto reduzido não é único em Vespoidea (BROTHERS; CARPENTER 1993), já que também ocorre em Chalcidoidea. Na filogenia de Brothers e Carpenter (1993), as formigas aparecem como grupo-irmão de Scoliidae + Vespidae.

Com a filogenia interna de Aculeata através de genes, Pilgrim et al. (2008) confirmam a monofilia de Aculeata, Chrysidoidea e Apoidea, mas estabelecem a não monofilia de Vespoidea, propondo várias superfamílias e sugerindo a proximidade entre apoideos e formigas, uma relação nova e inesperada. Esta mesma relação reaparece na análise usando genomas (filogenômica) de Johnson et al. (2013), onde se corrobora a relação entre apoideos e formigas. Da proposta de Brothers (1975 e seguintes), apenas Scolliidae permanece 
próximo, com Vespidae em uma posição muito distante. Estas relações, sendo estáveis, são muito importantes não apenas para buscar grupos externos adequados, como também para estabelecer cenários plausíveis sobre os primeiros passos na evolução das formigas. Um ancestral entre formigas e grupos vizinhos então já não consiste de uma vespa ectoparasitoide (de acordo com a filogenia de Brothers), mas em uma vespa que cava ninhos no solo, o qual aprovisiona de uma vez ou gradativamente, com presas, como ocorre em Ampulicidae e Sphecidae, as quais são famílias de apoideos basais. Este cenário pode tornar mais plausível a recente proposta das primeiras formigas habitantes do solo, originando posteriormente as linhagens que colonizaram a serapilheira e as árvores (LUCKY et al., 2013). Vale notar que a maioria das formigas poneroides é habitante do solo e apenas uma pequena parte é arbórea. Também vale lembrar o caso de Cariridris bipetiolata, um himenóptero fóssil da Formação Santana brasileira do Cretáceo, o qual foi originalmente descrito como uma formiga (BRANDÃO et al., 1990), considerada como ampulicídeo (VERHAAGH, 1996), depois como aculeado desconhecido (GRIMALDI et al., 1997) e novamente como formiga, mas depois não como uma formiga (BOLTON, 2014).

Atualmente são conhecidas 16 subfamílias de formigas viventes, todas monofiléticas. Esse número se baseia em argumentos de morfologia (BOLTON, 2003) e filogenia usando morfologia ou genes (BARONI URBANI et al., 1992; SAUX et al., 2004; MOREAU et al., 2006; BRADY et al., 2006; WARD et al., 2014).

\section{Filogenias baseadas em morfologia}

Desde a primeira síntese importante e moderna da classificação das formigas (BROWN, 1954), as formigas caçadoras constituíram uma subfamília mais ou menos estável, Ponerinae, definida em parte por atributos de morfologia externa. É nesta publicação (BROWN, 1954) que se tenta a primeira síntese crítica de divisão interna e Formicidae, na qual o autor propõe dois grandes agrupamentos, o complexo poneroide e o complexo formicoide. Brown (1954) posiciona Ponerinae no primeiro complexo (em um conceito distinto do atual), próxima a Cerapachyinae, e coloca Myrmicinae e as formigas-de-correição (Dorylinae s.l.). Uma proposta muito parecida pode ser vista em Wilson et al. (1967), onde se diferenciam os poneríneos primitivos (Amblyoponini) dos avançados e sugere que as formigas-de-correição surgiram de um grupo poneroide. Uma árvore muito semelhante é proposta por Taylor (1978), que sugere como caráter diagnóstico do complexo poneroide a fusão entre tergo e esterno do IV segmento abdominal. Um caráter que foi considerado de muito peso, mas que teve sua importância reavaliada com descobertas posteriores.

Brown (1958, 1960, 1975), em uma série de publicações que tratam não apenas de taxonomia alfa, mas também de níveis superiores, se propôs a reclassificar as formigas, começando pelas poneríneas. Em um destes trabalhos, Brown (1975) funde Cerapachyinae com Ponerinae, convertendo esta subfamília em uma das maiores e mais conspícuas entre as formigas. Mesmo com a biologia variada, muitas formigas desta subfamília compreendiam espécies com hábitos principalmente carnívoros, de onde se cunhou o nome "caçadoras", ainda que hoje saibamos que algumas são onívoras e que existam predadoras em outras linhagens de formigas (HÖLLDOBLER; WILSON 1990). O peso específico das ideias de Brown, taxonomista de linhagem pura, versado na escola evolutiva, entre os estudiosos de formigas é evidente desde seus ensaios iniciais de 1954 até o início do século XXI. Brown, contudo, eventualmente repensou a definição das poneríneas, além de suas relações com outras formigas, mas não pôde publicar suas ideias antes de falecer. Roberto Keller (2011) resgatou e resumiu claramente estas ideias posteriores de William Brown Jr. Com respeito ao caráter parafilético de Ponerinae sensu Brown. Este autor mostra claramente que seu complexo poneroide deve excluir Leptanillinae, Dorylinae s.l., Myrmicinae e, inclusive, Cerapachyinae.

O passo decisivo seguinte na exploração da filogenia interna das formigas foi dado por Baroni Urbani et al. (1992) ao usar formalmente a cladística e uma abundante quantidade de caracteres morfológicos. Seus grupos externos foram os vespídeos e os bradinobenídeos. Um grande clado desta filogenia corresponde aproximadamente ao complexo poneroide referido mais acima, com a exclusão de Myrmicinae, que passa ao outro clado, como grupo-irmão de Pseudomyrmecinae e Myrmeciinae. Leptanillinae e Cerapachyinae são excluídos de Ponerinae (que fica como grupo-irmão de Apomyrminae). Uma importante consequência desta filogenia é a delimitação das formigas-decorreição (Dorylinae s.l.) como grupo claramente 
monofilético, com a inclusão de Cerapachyinae e Leptanilloidinae. Ponerinae agora fica circunscrito ao conceito proposto por Brown (p.e. 1958, 1960, 1975), com a exclusão de Cerapachyinae e Leptanilloidinae. Os autores caracterizam Ponerinae como as formigas que possuem fusão tergo-esternal do quarto segmento abdominal e pré-escleritos longos do quarto segmento abdominal (também em Cerapachyinae e Myrmeciinae). Os autores também advertem sobre o grande grau de polimorfismo nesta subfamília.

Lattke (1994) explora a monofilia e relações das formigas da então tribo Ectatommini usando caracteres de morfologia e análise cladística. Este autor revive as tribos Paraponerini e Proceratiini e limita o conceito de Ectatommini a Ectatomma, Gnamptogenys, Rhytidoponera, Heteroponera e Acanthoponera. Keller (2000) faz alguns ajustes e correções a esta proposta, ainda que em essência as conclusões do primeiro autor se mantêm. Com estas contribuições ficou claro que a delimitação de Ponerinae no sentido então aceito era instável e seria necessário explorar mais caracteres, incluindo moleculares, para buscar as relações de todos os grupos incluídos Ponerinae.

Em uma obra fundamental para a classificação dos formicídeos, Bolton (2003) publica uma sinopse e classificação na qual propõe um agrupamento denominado formigas poneromorfas para as subfamílias Amblyoponinae, Ectatomminae, Heteroponerinae, Paraponerinae, Ponerinae e Proceratiinae. Leptanillinae e Apomyrminae passam ao grupo leptanilomorfo e Cerapachyinae ao grupo dorilomorfo. Apesar de oferecer uma diagnose para este agrupamento informal, não se postula nenhuma sinapomorfia para ele. Esta dificuldade é reconhecida pelo autor que prefere ao menos separá-las de outros grupos mais bem resolvidos. Felizmente, para cada uma das subfamílias que constituem as poneromorfas, Bolton (2003) propõe apomorfias morfológicas, com a exceção das heteroponeríneas, as quais agora saem de Ectatomminae.

Keller (2011) estudou comparativamente a morfologia externa (exoesqueleto) das formigas para realizar uma proposta em filogenia com ênfase nas formigas poneromorfas. Seu trabalho se destaca pelo esforço em definir os caracteres estudados e seus estados de maneira rigorosa e complementada por imagens de Microscópio Eletrônico de Varredura. O principal resultado é que as formigas poneromorfas formam um agrupamento parafilético, pois também incluem dorilomorfo, lepitanilomorfos e myrmicomorfos. Ainda que esta análise concorde com estudos prévios quanto à monofilia das subfamílias existentes e questiona a monofilia dos poneromorfos, ela não coincide com outros agrupamentos como sugerem as filogenias baseadas em genes (ver próxima seção). Os resultados sofreram com o número elevado de homoplasias, com árvores alternativas no que se refere às relações entre as poneromorfas e os três clados antes mencionados. A posição de Paraponera foi particularmente difícil de estabelecer já que em algumas árvores o gênero aparece como grupo-irmão das mirmecomorfas e proceratíneas, ou das heteroponeríneas e ectatommíneas.

\section{Filogenias baseadas em genes}

As análises moleculares recentes desenvolvidas para formigas (MOREAU et al., 2006; BRADY et al., 2006; RABELING et al., 2008; SCHMIDT, 2013; MOREAU; BELL, 2013) mostram um grupo claramente monofilético, o clado formicoide, o qual pode ser dividido em três subclados. O primeiro deles é o dorilomorfo, o qual inclui as formigas-de-correição, com as Dorylinae, Leptanilloidinae, "Cerapachyinae" e outros grupos do Velho Mundo. O segundo clado compreende Pseudomyrmecinae, Dolichoderinae e duas subfamílias mais do Velho Mundo. O terceiro clado inclui Formicinae, Myrmicinae e Ectatomminae + Heteroponerinae. $\mathrm{O}$ resto de formigas fica, infelizmente, sem uma resolução clara. Em Brady et al. (2006) aparecem as formigas do clado poneroide como grupo-irmão do clado formicoide, embora Ward, com mais realismo, sugere que seja melhor representar as formigas poneroides como um grado ou grupo parafilético (Figura 1.1 em WARD, 2010). De acordo com as árvores mencionadas, os poneromorfos ficam divididos em diferentes clados, sem qualquer condição de representar um grupo natural. $\mathrm{O}$ mesmo ramo de poneroides não tem sustentação contundente (WARD, 2010) dado que as relações internas e entre estas subfamílias e as demais ficam sem resolução.

Schmidt (2013) realiza a primeira filogenia molecular da subfamília Ponerinae no sentido estrito, corroborando a monofilia da subfamília e finalmente obtém sucesso na resolução das relações entre alguns de seus componentes. Seus resultados também sugerem uma relação de grupo-irmão de Ponerinae e Agroecomyrmecinae, uma conclusão 
que coincide com os resultados de Moreau et al. (2006) e Brady et al. (2006). Contudo, isto contradiz os resultados de Rabeling et al. (2008) e alguns dos achados de Brady et al. (2006), os quais postulam uma relação de grupo-irmão entre poneríneas e o grupo formicoide. Adicionalmente, o clado ectaheteromorfo aparece como grupo-irmão de Formicinae + Myrmicinae. Em seu estudo da filogenia e evolução das formigas sob a hipótese dos trópicos como "museu" ou "berço" da diversificação destes insetos, Moreau; Bell (2013) inferem a filogenia molecular de representantes de, até então, 21 subfamílias. Estes autores estabelecem uma idade entre 158 e 139 milhões de anos e 10 períodos de mudanças significativas na diversificação das formigas. Em comparação com as propostas mencionadas acima, o clado Ectatomminae + Formicinae aparece como grupo-irmão de Myrmicinae, e Formicinae como grupo-irmão destas subfamílias. Os poneroides formam uma politomia com os formicoide e Martialinae, e Leptanillinae aparece como grupo-irmão de todas as formigas.

\section{Classificação}

Como consequência dos estudos recentes em filogenia de formigas, a antiga subfamília Ponerinae (BOLTON, 1994) ficou fragmentada e dividida em várias subfamílias: Amblyoponinae, Ectatomminae, Heteroponerinae, Paraponerinae, Ponerinae s. str. e Proceratiinae. Já se consideravam subfamílias independentes Cerapachyinae e Leptanilloidinae (agora ambas no grupo das formigas-de-correição).

O Quadro 7.I lista os clados e subfamílias de formigas segundo o estudo de Brady et al. (2006). O Quadro 7.II relaciona o número de gêneros e espécies para cada uma das subfamílias de formigas poneromorfas no mundo e na Região Neotropical. Como se pode ver, as subfamílias que compreendiam Ponerinae ou o grupo das poneromorfas

QUADRO 7.I - Sinopses das formigas caçadoras (grado poneroide e subclado ectaheteroponeríneo) na Região Neotropical). Em cada táxon é dado o número de espécies e distribuição. Tomado de Jiménez et al. (2008) e com as mudanças propostas em Schmidt e Shattuck (2014). O número de espécies compreende as descritas recentemente em Mackay e Mackay (2010).

\begin{tabular}{|l|}
\hline Agrupamento Poneroide (Parafilético) \\
\hline Subfamília Amblyoponinae (17) \\
Tribo Amblyoponini \\
Stigmatomma Roger, 1859 - 12, Neotrópico \\
Prionopelta Mayr, 1866 - 4, Neotrópico \\
Incertae sedis: \\
Paraprionopelta Kusnezov, 1955 - 1, Argentina \\
Subfamília Paraponerinae (1) \\
Tribo Paraponerini \\
Paraponera F. Smith, 1858 - 1, Neotrópico \\
Subfamília Proceratiinae (25) \\
Tribo Proceratiini \\
Discothyrea Roger, $1863-7$, Neotrópico \\
Proceratium Roger, 1863 - 15, Neotrópico \\
Tribo Probolomyrmecini \\
Probolomyrmex Mayr, 1901 - 3, Neotrópico \\
Subfamília Ponerinae (275) \\
Tribo Ponerini \\
Anochetus Mayr, 1861 - 25, Neotrópico \\
Belonopelta Mayr, 1870 - 2, Neotrópico \\
Centromyrmex Mayr, 1866 - 3, Neotrópico \\
Cryptopone Emery, $1893-3$, Neotrópico \\
Dinoponera Roger, 1861 - 8, Sudamérica \\
Hypoponera Santschi, 1938 - 36, Neotrópico \\
\hline
\end{tabular}

\begin{tabular}{|l|}
\hline Agrupamento Poneroide (Parafilético) \\
\hline Leptogenys Roger, 1861 - 80, Neotrópico \\
Mayaponera Schmidt; Shattuck, 2014 - 1, Neotrópico \\
Neoponera Emery, 1901 - 68, Neotrópico \\
Odontomachus Latreille, 1804 - 25, Neotrópico \\
Pachycondyla F. Smith, 1858 - 13, Neotrópico \\
Pseudoponera Emery, 1900 - 5, Neotrópico \\
Rasopone Schmidt; Shattuck, 2014 - 11, Neotrópico \\
Simopelta Mann, 1922 - 21, Neotrópico \\
Thaumatomyrmex Mayr, 1887 - 12, Neotrópico \\
Tribo Platythyreini \\
Platythyrea Roger, 1863 - 8, Neotrópico \\
Clado Formicoide \\
Grupo Ectatomminoide \\
Subfamília Ectatomminae (112) \\
Tribo Ectatommini \\
Ectatomma F. Smith, 1858 - 15, Neotrópico \\
Gnamptogenys Roger, 1863 - 90, Neotrópico \\
Tribo Typhlomyrmecini \\
Typhlomyrmex Mayr, 1862 - 8, Neotrópico \\
Subfamília Heteroponerinae (16) \\
Tribo Heteroponerini \\
Acanthoponera Mayr, 1882 - 4, Neotrópico \\
Heteroponera Mayr, 1877 - 12, Neotrópico \\
\hline
\end{tabular}


QUADRO 7.II - Número de gêneros e espécies de formigas poneromorfas no Mundo (BOLTON, 2014) e a Região Neotropical

\begin{tabular}{|l|r|r|r|r|}
\hline & \multicolumn{2}{|c|}{ Gêneros } & \multicolumn{2}{c|}{ Espécies } \\
\hline & \multicolumn{1}{|c|}{ Mundo } & \multicolumn{1}{c|}{ Neotrop } & \multicolumn{1}{c|}{ Mundo } & \multicolumn{1}{c|}{ Neotrop } \\
\hline Amblyoponinae & 13 & 3 & 125 & 17 \\
\hline Paraponerinae & 1 & 1 & 1 & 1 \\
\hline Proceratiinae & 3 & 3 & 145 & 25 \\
\hline Ponerinae & 47 & 15 & 1195 & 321 \\
\hline Ectatomminae & 4 & 3 & 264 & 114 \\
\hline Heteroponerinae & 3 & 2 & 24 & 16 \\
\hline Total & $\mathbf{7 1}$ & $\mathbf{2 7}$ & $\mathbf{1 7 5 4}$ & $\mathbf{4 9 4}$ \\
\hline
\end{tabular}

ficaram dispersas. Já que os poneroides são um grupo parafilético (WARD, 2010, 2014) e não há evidências que permitam, por enquanto, resolver seu status ou relações, fica aberta a pergunta sobre qual nível taxonômico devemos usar para nos referirmos às formigas caçadoras.

Seguindo Bolton (2003, 2015), e Schmidt; Shattuck (2014), o Quadro 7.I relaciona seis subfamílias, 10 tribos e 24 gêneros, que abrangem aproximadamente 460 espécies. De longe, as subfamílias Ponerinae e Ectatomminae são as maiores em táxons contidos, assim como as tribos Ponerini e Ectatommini, graças ao fato de que compreendem gêneros com muitas espécies como Gnamptogenys, Hypoponera, Neoponera, Odontomachus e Leptogenys. Por outro lado, alguns dos táxons menos conhecidos de poneroides compreendem gêneros raramente coletados $\mathrm{e}$ geralmente com poucas espécies descritas como Probolomyrmex, Thaumatomyrmex, Platythyrea ou Discothyrea. Por outro lado, as formigas caçadoras compreendem gêneros taxonomicamente complexos, como Hypoponera e Discothyrea, os quais compreendem formigas pequenas, monótonas com poucos ou nenhum caráter que permita delimitar espécies facilmente ou com algum grau de confiança.

\section{Conclusões e perspectivas}

A delimitação atual e compreensão dos diversos aspectos das formigas caçadoras poneroideas começou com as propostas de William Brown e outros autores desde a década de 1950. É a partir dos anos 90 que os métodos cladísticos oferecem hipóteses mais estáveis e sujeitas a críticas, com o delineamento de alguns grupos que posteriormente se fortaleceram como monofiléticos, como as formigas-de-correição (Dorylinae) ou o clado formicoide.
$\mathrm{O}$ que durante anos se concebeu como a subfamília Ponerinae, muito diversa tanto no número de táxons como em hábitos de vida, terminou fragmentando-se em várias subfamílias em diferentes clados. As poneríneas seguem sendo diversas, mas agora há maior coerência em seus hábitos de vida e crescente concordância quanto sua monofilia.

O par de subfamílias Ectatomminae + Heteroponerinae forma um grupo monofilético bem posicionado no subclado formicíneo-mirmicíneo dentro do clado formicoide. Contudo, não há consenso se estas subfamílias são o grupo-irmão de Formicinae + Dolichoderinae, ou de Myrmicinae. Em um estudo que se fundamente nos resultados de Moreau et al. (2006), Brady et al. (2006) e Rabeling et al. (2008), Moreau; Bell (2013) posicionam as ectaheteromorfas como grupo-irmão das mirmicíneas, ainda que sem suporte estatístico. Se este é de fato o caso, é interessante recordar a antiga proposta de Brown (1973), no sentido em que os ectatommíneos estão próximos ou filogeneticamente associados à Myrmicinae.

O restante dos grupos da antiga subfamília Ponerinae se dividiu em várias subfamílias, todas membros de um clado chamado poneroide, o qual inclui Agroecomyrmecinae (talvez como grupo-irmão de Paraponerinae) e exclui "Cerapachyinae", grupo sinonimizado sob Dorylinae (BRADY et al., 2014). Se os poneroides são um grupo monofilético e se são grupo-irmão dos formicoide ou se formam uma politomia com este grupo e Martialinae / Leptanillinae são assuntos pendentes.

Whitfield (2008) aponta que, devido a radiações rápidas antigas de muitas linhagens de insetos, a informação morfológica e/ou molecular foi apagada ou se tornou confusa de tal forma que perdemos as pistas para reconstruir filogenias de grupos basais ("stem groups"). Se as formigas se 
originaram há muito tempo (talvez na transição Jurássico-Cretáceo) em um período curto de diversificação explosiva, a clarificação das relações de seus grupos basais será difícil. As filogenias e estudos mais recentes (e.g. LUCKY et al., 2013) sugerem que as primeiras formigas eram habitantes do solo, para apenas depois conquistarem outros ambientes como a serapilheira e a vegetação. Os poneríneos estariam entre estas primeiras linhagens e seriam um grupo muito antigo, coexistindo com os dinossauros por um longo período de tempo e resistindo à extinção em massa da transição Cretáceo-Paleoceno (LAPOLLA et al., 2013).

Esta antiguidade não deve ser interpretada como indicadora de rusticidade: as formigas caçadoras são um grupo diverso, com amplas formas de vida e especializações surpreendentes. Se considerarmos as morfologias enigmáticas evidentemente muito especializadas de formigas formicoide fósseis com 100 milhões de anos (BARDEN; GRIMALDI, 2014; PERRICHOT, 2014), podemos apenas sonhar acordados com as formas que existiram em poneroides naquela época. Esta antiguidade combinada com a diversidade atual de morfologias e histórias de vida foram bem sucedidas em criar um grande interesse em sua biologia e comportamento (SCHMIDT; SHATTUCK, 2014).

\section{Agradecimentos}

Aos Drs. Rodrigo Feitosa (UFPR), Jacques Delabie (CEPLAC) e demais colegas do PRONEX, pelo convite para a participação no presente livro sobre formigas caçadoras. A Rodrigo Feitosa, pela tradução e observações.

\section{Literatura citada}

BARDEN, P.; GRIMALDI, D. A diverse ant fauna from the mid-Cretaceous of Myanmar (Hymenoptera: Formicidae). PLoS One, 9(4):e93627, 2014.

BARONI URBANI C., B. BOLTON; P.S. WARD. The internal phylogeny of ants (Hymenoptera: Formicidae). Systematic Entomology, 17:301-329, 1992.

BOLTON B. Identification Guide to the Ant Genera of the World. Harvard University Press, Cambridge, Massachusetts, E.E.U.U. 1994. 222 p.

BOLTON B. Synopsis and classification of Formicidae. Memoirs of the American Entomological Institute, 71: 1-370, 2003.
BOLTON, B. An online catalog of the ants of the world. Disponible en http://antcat.org. (visto [3.iv.2015])

BRADY S.G., SCHULTZ T.R., FISHER B.L.; WARD P.S. Evaluating alternative hypothesis for the early evolution and diversification of ants. Proceedings of the National Academy of Sciences of the Unitd States of America, 103(48):18172-18177, 2006.

BRADY, S. G.; FISHER, B. L.; SCHULTZ, T. R.; WARD, P. S. The rise of army ants and their relatives: diversification of specialized predatory doryline ants. BMC Evolutionary Biology, 14:93, 14 p., 2014.

BRANDÃO, C. R. F.; MARTINS-NETO, R. G. [Untitled. Cariridris Brandão; Martins-Neto, new genus. Cariridris bipetiolata Brandão; Martins-Neto, new species.]. Pp. 201-202 en: Brandão, C. R. F.; MartinsNeto, R. G.; Vulcano, M. A. 1990. The earliest known fossil ant (first southern hemisphere Mesozoic record) (Hymenoptera: Formicidae: Myrmeciinae). Psyche (Cambridge), 96:195-208, 1990 (“1989”).

BROTHERS, D. J. Phylogeny and Classification of the Aculeate Hymenoptera, With Special Reference to the Mutillidae. University of Kansas Science Bulletin, 50, 483-648, 1975.

BROTHERS, D. J.; CARPENTER, J. M. Phylogeny of Aculeata: Chrysidoidea and Vespoidea (Hymenoptera). Journal of Hymenoptera Research, 2:227-304, 1993.

BROWN W. L. JR. Remarks on the internal phylogeny and subfamily classification of the family Formicidae. Insectes Sociaux, 1:21-31, 1954.

Brown W. L. Contributions toward a reclassification of the Formicidae. II. Tribe Ectatommini (Hymenoptera). Bulletin of the Museum of Comparative Zoology, 118: 175-362, 1958.

BROWN, W.L. JR. Contributions toward a reclassification of the Formicidae. III. Tribe Amblyoponini (Hymenoptera). Bulletin of the Museum of Comparative Zoology, 122 (4), 145-230, 1960.

BROWN, W.L. JR. A comparison of the Hylean and Congo-West African rain forest ant faunas. In: Meggers, B.J., Ayensu, E.S.; Duckworth, W.D. (Eds.), Tropical Forest Ecosystems in Africa and South America: a Comparative Review. Smithsonian Institution Press, Washington, D.C. 1973. p. 161-185.

BROWN W. L. Contributions toward a reclassification of the Formicidae. V. Ponerinae, tribes Platythyreini, Cerapachyini, Cylindromyrmecini, Acanthostichini, and Aenictogitini, Search Agriculture, 15: 1-115, 1975. 
GRIMALDI, D.; AGOSTI, D. A formicine in New Jersey Cretaceous amber Hymenoptera: Formicidae) and early evolution of the ants. Proceedings of the National Academy of Sciences of the United States of America, 97, 13 678-13 683, 2000.

GRIMALDI, D.; AGOSTI, D.; CARPENTER, J. M. New and rediscovered primitive ants (Hymenoptera: Formicidae) in Cretaceous amber from New Jersey, and their phylogenetic relationships. American Museum Novitates, 3208:1-43, 1997.

HERATY, J.; RONQUIST, F.; CARPENTER, J. M.; HAWKS, D.; SCHULMEISTER, S.; DOWLING, A. P.; MURRAY, D.; MUNRO, J.; WHEELER, W. C.; SCHIFF, N.; SHARKEY, M. Evolution of the hymenopteran megaradiation. Molecular Phylogenetics and Evolution, 60:73-88, 2011.

HÖLLDOBLER B.; E.O. WILSON. The Ants. Belknap Press, Cambridge, Massachusetts, E.E.U.U. 1990. p. 732.

JIMÉNEZ, E., FERNÁNDEZ, F., ARIAS, T.M.; LOZANO-ZAMBRANO, F.H. (Eds.), Sistemática, Biogeografía y Conservación de las Hormigas Cazadoras de Colombia. Instituto de Investigación de Recursos Biológicos Alexander von Humboldt, Bogotá, pp. 285-328.

JOHNSON, B. R.; BOROWIEC, M. L.; CHIU, J. C.; LEE, E. K.; ATALLAH, J.; WARD, P. S. Phylogenomics resolves evolutionary relationships among ants, bees, and wasps. Current Biology, 23:2058-2062, 2013.

KELLER R. Cladistics of the tribe Ectatommini (Hymenoptera: Formicidae): a reappraisal. Insect Systematics and Evolution, 31: 59-69, 2000.

KELLER, R. A. A phylogenetic analysis of ant morphology (Hymenoptera: Formicidae) with special reference to the poneromorph subfamilies. Bulletin of the American Museum of Natural History, 355:1-90, 2011.

LAPOLLA, J. S.; DLUSSKY, G. M.; PERRICHOT, V. Ants and the fossil record. Annual Review of Entomology, 58:609-630, 2013.

LATTKE, J. Phylogenetic relationships and classification in the Ectatommini, (Hymenoptera: Formicidae). Entomologica Scandinavica, 25:105-119, 1994.

LUCKY, A.; TRAUTWEIN, M. D.; GUÉNARD, B.; WEISER, M. D.; DUNN, R. R. Tracing the rise of the ants - out of the ground. PLoS One, 8(12): e84012, 2013.

MACKAY W. P.; E. The Systematics and Biology of the New World Ants of the Genus Pachycondyla (Hymenoptera: Formicidae). Edwin Mellen Press, 2010. p. 649.
MOREAU C.S., C.D. BELL, R. VILA, S.B.

ARCHIBALD; N.E. PIERCE. Phylogeny of the ants: Diversification in the age of Angiosperms. Science, 312:101-104, 2006.

MOREAU, C.S.; BELL, C.D. Testing the museum versus cradle tropical biological diversity hypothesis: phylogeny, diversification, and ancestral biogeographic range evolution of the ants. Evolution, 67: 2240-2257, 2013

PERRICHOT, V. A new species of the Cretaceous ant Zigrasimecia based on the worker caste reveals placement of the genus in the Sphecomyrminae (Hymenoptera: Formicidae). Myrmecological News, 19:165-169, 2014

PILGRIM, E. M., VON DOHLEN, C. D.; PITTS, J. P. Molecular phylogenetics of Vespoidea indicate paraphyly of the superfamily and novel relationships of its component families and subfamilies. Zoologica Scripta, 37: 539-560, 2008.

RABELING C., J. BROWN; M. VERHAAGH. Newly discovered sister lineage sheds light on early ant evolution. Proceedings of the Natural Academy of Science of the United States of America, 105:1491314917, 2008.

RONQUIST, F.; RASNITSYN, A. P.; ROY, A.; ERIKSSON, K.; LINDGREN, M. Phylogeny of the Hymenoptera: a cladistic reanalysis of Rasnitysn's (1988) data. Zoologica Scripta, 28:13-50, 1999.

SAUX C., FISHER B. L.; SPICER G. S. Dracula ant phylogeny as inferred by nuclear 28S rDNA sequences and implications for ant systematic (Hymenoptera: Formicidae: Amblyoponinae) Molecular Phylogenetics and Evolution, 33: 457-468, 2004.

SCHMIDT, C. Molecular Phylogenetics of ponerine ants (Hymenoptera: Formicidae: Ponerinae). Zootaxa, 3647:201-150, 2013.

SCHMIDT C.; SHATTUCK S.O. The higher classification of the ant subfamily Ponerinae (Hymenoptera: Formicidae: Ponerinae) with a review of ponerine eology and behavior. Zootaxa, 3817:1-242, 2014.

TAYLOR, R. W. Nothomyrmecia macrops: a livingfossil ant rediscovered. Science (Washington, D. C.), 201:979-985, 1978a.

TAYLOR, R. W. Bloody funny wasps! Speculations on the evolution of eusociality in ants. Memoirs of the American Entomological Institute, 80:580-609, 2007. 
VERHAAGH, M. Warum die älteste bekannte Ameise keine ist (Hymenoptera). Beiträge der Hymenopterologen-Tagung in Stuttgart, 1996:11, 1996.

WARD P.S. Taxonomy, phylogenetics and evolution, p. 3-17 en: L. Lach, C. Parr; K. Abbott, eds., Ant Ecology, Oxford University Press, 2010.

WARD, P. S.; BRADY, S. G.; FISHER, B. L.; SCHULTZ, T. R. The evolution of myrmicine ants: phylogeny and biogeography of a hyperdiverse ant clade (Hymenoptera: Formicidae). Systematic Entomology, (2014):1-21, 2014.
WARD, P. S. The phylogeny and evolution of ants. Annual Review of Ecology, Evolution, and Systematics, 45:2.1-2.21, 2014.

WHITFIELD J.M.; K.M. KJER. Ancient Rapid Radiations of Insects: Challenges for Phylogenetic Analysis. Annual Review of Entomology, 53:449-472, 2008.

WILSON E. O., CARPENTER J. M. Y BROWN JR. W. L. The first Mesozoic ants. Science, 157:1038-1040, 1967. 